\title{
A METHOD TO SELECT REPRESENTATIVE ROCK SAMPLES FOR DIGITAL CORE MODELING
}

\author{
WEI LIN $, *, \dagger, \uparrow$, ZHENGMING YANG $, *, \dagger, \uparrow, \|$ XIZHE LI, ${ }^{*, \dagger, \dagger}$ \\ JUAN WANG,$\S$ YING HE,,, GUOMING WU, $,, \dagger, \ddagger$ \\ SHENGCHUN XIONG ${ }^{\dagger, \ddagger}$ and YUNYUN WEI, $, \dagger, \ddagger$ \\ ${ }^{*}$ University of Chinese Academy of Sciences \\ Beijing 100049, P. R. China \\ ${ }^{\dagger}$ Institute of Porous Flow and Fluid Mechanics \\ Chinese Academy of Sciences, Langfang, Hebei 06500\%, P. R. China \\ ${ }^{\ddagger}$ Research Institute of Petroleum Exploration and Development \\ Beijing 100083, P. R. China \\ ${ }^{\S}$ School of Energy Resources, China University of Geosciences \\ Beijing 100083, P. R. China \\ Iucaslinwei@126.com \\ "yzhm69@petrochina.com.cn
}

Received January 25, 2017

Revised April 3, 2017

Accepted April 18, 2017

Published May 29, 2017

\begin{abstract}
X-ray computed tomography $(\mathrm{CT})$ scanning method is the most accurate method to construct digital core, which can reflect the microscopic pore structure of real cores; therefore, it is widely used and researched by experts and scholars all over the world. However, there are few
\end{abstract}

\footnotetext{
${ }$ Corresponding author.

This is an Open Access article published by World Scientific Publishing Company. It is distributed under the terms of the Creative Commons Attribution 4.0 (CC-BY) License. Further distribution of this work is permitted, provided the original work is properly cited.
} 
reports about how to select the CT scan core samples at present, and the current practice is to make CT scan samples by visually observing rocks or core columns to select a region that is considered representative or interesting, which can lead to a large difference between the selected sample and the whole rock and a digital core that cannot represent the real rock as a whole. In order to construct the digital cores that can reflect the whole rock structure and reservoir properties, combining with fractal theory, a scientific and reasonable method was proposed to select representative rock samples for digital core modeling. First of all, a core column is scanned by X-ray CT at a certain resolution and CT gray scale images are obtained and stored in the order of scan. Secondly, the fractal dimension (FD) of each image is calculated by box-counting method, and the calculated porosity of each image is achieved by the existing formula. Then, according to the size of the digital core to be constructed, the CT gray scale images are grouped, and the average FD and the average porosity of each combination are calculated by the derived equations. Finally, based on the proposed criteria the best image combination is selected and the preferred sample is determined accordingly. At the same time, a facile experiment was conducted to test the effectiveness of this method. The experimental results show that there are some errors between the subjectively selected cores and the long core in terms of permeability and porosity, and the petrophysical parameters of the core selected by the proposed method are close to those of the long core; as a consequence, the validity of this method was verified and it is feasible and practical to select the representative rock samples for digital core modeling by this method.

Keywords: Digital Core; Fractal Theory; X-ray CT Scan; Representative Rock Sample.

\section{INTRODUCTION}

As a key means to carry out the numerical simulation of rock physics, digital core technology has been widely studied by experts and scholars all over the world ${ }^{[14}$ At the same time, as the basis and key to research technologies of improving oil recovery in microscale, digital core technology has broad application prospects in the development of oil and gas field, such as the following aspects ${ }^{3}$ : (1) study on microscopic percolation mechanism and macroscopic conductivity prediction; (2) study on displacement mechanism and application effect evaluation of oil displacement agent; (3) simulation and prediction of reservoir production and (4) providing basis for the determination of technical policy boundary of oil field development.

Digital core construction methods are divided into two categories: physical experiment method and numerical reconstruction method 14 Physical experiment method, $\frac{5[6]}{5}$ as the name suggests, is a way to construct 3D digital cores using various kinds of petrophysical experimental equipments, such as scanning electron microscope, nuclear magnetic resonance (NMR), computed tomography (CT) scanner, etc. This method must directly or indirectly obtain 2D images of cores and then use image processing and mathematical methods to reconstruct 2D images of different sections into $3 \mathrm{D}$ digital cores. ${ }^{[]}$According to the difference of petrophysical experiments, physical experiment method is mainly divided into sequence imaging method,, 8 focus scanning method,, 90 and X-ray CT scanning method! $11 / 12$ Numerical reconstruction method 13 is mainly based on $2 \mathrm{D}$ information, such as rock casting thin slice and rock grain size data and so on, to extract the key information for constructing digital cores by image analysis and mathematical statistics method and then use mathematical algorithms for 3D reconstruction. According to the difference of algorithms, numerical reconstruction method is mainly divided into stochastic method and process method. In addition, different stochastic methods select different statistical characteristics as reconstruction constraint functions, so the stochastic method can be subdivided into Gauss field method, simulated annealing method, multi-point geostatistics method, sequential indicator simulation method and so on. 17

From the view of many digital core modeling methods, X-ray CT scan is the most accurate method of constructing digital core, which can reflect microscopic pore structure of real cores and is widely used at present! 147 The process of constructing 3D digital cores by X-ray CT can be divided into the following six steps $\$$ : 7 : (1) selection and preparation of sample; (2) sample X-ray CT 
scanning; (31) gray image filtering; (4) binarization of gray image; (5) smoothing processing of binary image and (6) analysis of representative volume element.

At present, the research on each step of the X-ray CT scanning method described above has been quite mature, except step (1). Furthermore, there are few reports about how to select the core CT scan target area; the current practice is to drill and produce a CT scan sample by visually observing the rock or core column to select a region that is considered representative or interesting, and the advantage of this practice is that it is simple and convenient and the disadvantage is that the sample selection is arbitrary; the selected sample does not necessarily represent the whole rock or core. 2/7/17 19 It is important to construct digital cores that truly reflect the characteristics of the reservoir or the rock as a whole, in an attempt to address the practical problems of oil field production through digital core technology. However, the CT scan resolution is inversely proportional to the size of the sample, and the diameter of rock sample is typically a few millimeters to several centimeters. ${ }^{[7]}$ In order to truly reflect the rock's overall structure and reservoir properties by such a small sample as much as possible, it is necessary to select the most representative rock sample to construct the digital core. Therefore, it is of great theoretical and practical significance to carry out the research on the method of selecting representative rock samples in digital core modeling.

Aiming at the problem of irregular selection of CT scan samples in digital core modeling and combining with the fractal theory, this paper establishes a scientific and reasonable method for selecting the target domain of digital core modeling.

\section{FRACTAL THEORY}

Fractal theory is a scientific method and theory formally proposed and established by Benoit B. Mandelbrot in 1975 to explore the complexity of objects. It was first born in the natural geometry and shows broad application prospects in many areas after nearly decades of development.20|21

Fractal is a kind of form in which the form of each component is similar to the whole in a certain way. It has self-similarity and scale invariance and is an effective tool for describing non-smooth and irregular geometries in natural and non-linear systems, which can be used to simulate many natural phenomena. 22

A large number of studies have shown that the pore structure of reservoir rock has typical fractal characteristics. Fractal dimension (FD) can be used to describe the fractal characteristics of pore distribution. Additionally, it is a new method different from the conventional method that fractal geometry theory is used to analyze and study the pore structure characteristics of reservoir rocks, which has been and will continue to be in-depth and widely used in geological analysis. $23+32$

\subsection{Calculation of FD by Box-Counting Method}

In CT gray image, the pixel gray value is in the range of $[0,255]$, as shown in Fig. 1] In the $3 \mathrm{D}$ coordinate system, the gray value of $2 \mathrm{D}$ image is actually a

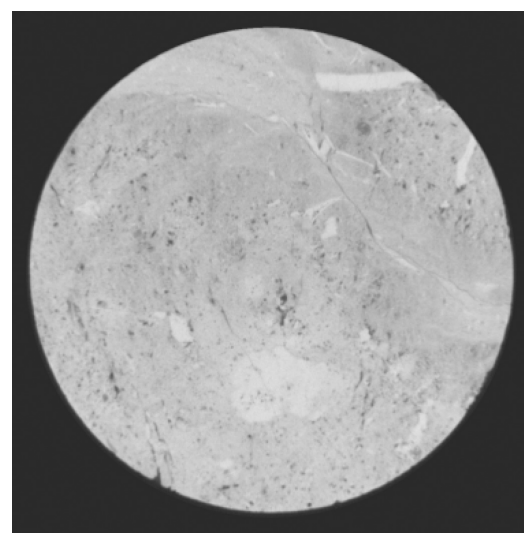

(a) CT gray image

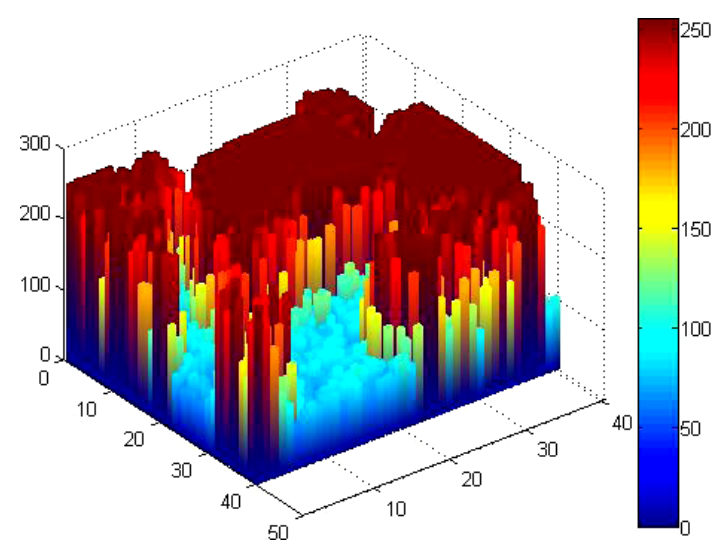

(b) Gray histogram of CT gray image (local)

Fig. 1 CT gray image and gray histogram. 
gray surface $[x, y, z(x, y)]$, where $z(x, y)$ is the gray value of position $[x, y]$. Therefore, the gray scale of the image will be reflected in the roughness of the gray scale surface and the use of different scales to measure the surface; the resulting dimension is the FD of the image gray scale surface.

The algorithm of box-counting method (BCM) is as follows. 20.21

Assuming that the image size is $M * N$ pixels, an $S * S$ grid is used to cover the image completely, $S$ is an integer, $M / 2 \geq S>1$ and the division ratio $r=S / M$. On each grid of size $S * S$, a square box of size $S * S * H$ is used to divide the $3 \mathrm{D}$ gray space. Suppose that in the grid column or box $(i, j)$, the minimum and maximum gray values of the image fall in the $k$ th and $f$ th boxes, respectively, and the number of boxes $n_{r}$ required to completely cover the gray values in the grid $(i, j)$ is as follows:

$$
n_{r}=f-k+1 \text {. }
$$

In Eq. (1), $k$ and $f$ are the numbers of the boxes that the minimum and maximum gray levels of the image in the $(i, j)$ th grid fall in, respectively. Further, the number of boxes $N_{r}$ required to cover the entire image can be obtained as follows:

$$
N_{r}=\sum n_{r}
$$

By changing the value of $S$, repeating the above process, we will get a new set $\left(N_{r}, r\right)$.

Then, according to the formula $\log N_{r}=D \log$ $(1 / r)+\log \kappa$, where $D$ is the box dimension and $\kappa$ is the constant. $\left[\log N_{r}, \log (1 / r)\right]$ is linearly fitted using the least-squares method, and the slope value of the fitted line is the fractal box dimension $D$.

\subsection{Relationship Between FD and Porosity}

In the scale-free interval, the cumulative size distribution of pores should follow the following scaling law 2426:

$$
N(L \geq \lambda)=\left(\frac{\lambda_{\max }}{\lambda}\right)^{D_{\mathrm{f}}},
$$

where $N$ is the cumulative number of pores, $\lambda$ is the pore diameter, $\lambda_{\max }$ is the maximum pore diameter, $L$ is the measurement scale and $D_{\mathrm{f}}$ is the FD.

Based on Eq. (3), Yu and Li24 derived a relation for FD for pore volume of saturated/single-phase porous media. This relation analytically relates the FD $D_{\mathrm{f}}$ to porosity and microstructural parameters, $\lambda_{\max }$ and $\lambda_{\min }$, and the relation is given by

$$
\phi=\left(\frac{\lambda_{\min }}{\lambda_{\max }}\right)^{d_{\mathrm{E}}-D_{\mathrm{f}}},
$$

where $d_{\mathrm{E}}$ is the Euclidean dimension and $d_{\mathrm{E}}=2$ and 3 in the 2D and 3D spaces, respectively, $\lambda_{\max }$ and $\lambda_{\min }$ are the maximum and minimum pore diameters and $\phi$ is the porosity.

\subsection{Calculation of the Parameters $D_{\mathrm{f}}, \lambda_{\min }$ and $\lambda_{\max }$}

In this paper, $d_{\mathrm{E}}=2$, because the single image belongs to the 2D study body. According to Eq. (4), it is necessary to calculate $D_{\mathrm{f}}, \lambda_{\max }$ and $\lambda_{\min }$ before obtaining the porosity $\phi$. The edge detection technique is used to process the CT gray image, which is realized by self-compiled MATLAB version of the microscopic pore result edge extraction sub-function, calling edge function to achieve the recognition and extraction of pixels with dramatic changes in gray value. The program input is CT gray image, and the output includes edge pixel coordinates and the binary image with edge information. In addition, due to the complexity of the real pore shape, it is difficult to obtain the characteristic parameters, microscopic pores are regarded as circular areas equal to their areas, so as to obtain the equivalent diameter $\lambda$. The program reads the CT gray image in batches. After edge detection, it statistically outputs the number of microscopic pores and the equivalent diameter of each image to the specified Excel file.

It can be found from Eq. (3) that in the scale-free interval, the cumulative number of pores $N$ and the equivalent diameter $\lambda$ follow the power-law relation; the expression in the logarithmic coordinates is as follows:

$$
\ln [N(L \geq \lambda)]=-D_{\mathrm{f}} \ln \lambda+D_{\mathrm{f}} \ln \lambda_{\max } .
$$

Obviously, the cumulative number of pores $N$ and the equivalent diameter $\lambda$ follow the linear relationship, and the slope value of the line is the FD $D_{\mathrm{f}}$. In order to accurately divide the linear segment, we employed a linear decision method. Specifically, the principle of this method is that the second derivative of the straight line is zero and that of the curve is not zero. Based on this principle, we wrote the derivative solution and linear segment markup program by MATLAB. When a series of data pairs are entered, the program automatically calculates the second derivative of the curve. Setting a certain 
error value, the portion where the curve fluctuation is below the error value is divided into a scale-free segment and the corresponding data pairs will be recorded.

Using the linear decision method and the automated procedure, the accurate determination of the scale-free interval can be achieved and then the corresponding $D_{\mathrm{f}}, \lambda_{\min }$ and $\lambda_{\max }$ are obtained.

\section{METHOD DESCRIPTION}

The specific steps for selecting representative rock samples are as follows.

Step 1: First, the interesting core column in the study area is selected by visual inspection, and the core column is scanned by X-ray CT at an appropriate resolution. The length of the core column is $L$ and the spacing between the two CT slices is $d$, as shown in Fig. 2] A set of CT scan gray scale images is obtained, and the gray scale images are stored according to the correspondence relation of $\operatorname{pic} 1 \rightarrow \operatorname{slice} 1, \operatorname{pic} 2 \rightarrow \operatorname{slice} 2, \ldots, \operatorname{pic} N \rightarrow \operatorname{slice} N$.

Step 2: Then, the CT scan gray scale images are imported into the program written by $\mathrm{BCM}$ in Sec. 2.1, and the FD of the image gray scale surface of each image $D_{i}(i \in(1, N))$ is calculated separately and the results are imported into the specified Excel. Likewise, the CT scan gray scale images bulk into the automated program based on Sec. 2.3. $D_{\mathrm{f}}^{i}$, $\lambda_{\min }^{i}$ and $\lambda_{\max }^{i}(i \in(1, N))$ of each image are calculated and the results are imported into the specified Excel. Afterwards, $D_{\mathrm{f}}^{i}, \lambda_{\min }^{i}$ and $\lambda_{\max }^{i}$ obtained previously are imported into the program written in Sec. 2.2 to calculate the porosity of each image $\phi_{i}(i \in(1, N))$ and the results are imported into the specified Excel.

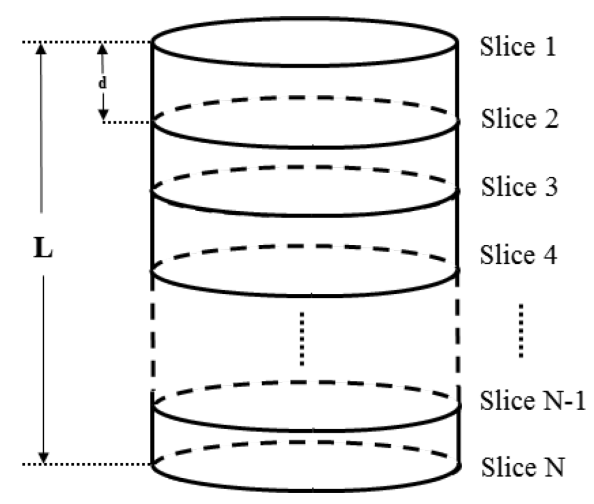

Fig. 2 Schematic diagram of core CT scan.
Step 3: Finally, according to the size of the digital core $l * l * l$ to be constructed, the number of $\mathrm{CT}$ scan images $n$ is determined, where $n<N, n * d \geq l$, the size of core column $L>l$. The $N-n+1$ kinds of image combinations are generated as follows:

$$
\begin{aligned}
I_{1} & =[\operatorname{pic} 1, \operatorname{pic} 2, \ldots, \operatorname{pic} n] \\
I_{2} & =[\operatorname{pic} 2, \operatorname{pic} 3, \ldots, \operatorname{pic}(n+1)], \ldots, I_{(N-n+1)} \\
& =[\operatorname{pic}(N-n+1), \operatorname{pic}(N-n+2), \ldots, \operatorname{pic} N]
\end{aligned}
$$

The average FD $\overline{D_{I_{j}}}$ and the average porosity $\overline{\phi_{I_{j}}}$ of each combination are calculated by the following equations, respectively:

$$
\begin{gathered}
\overline{D_{I_{j}}}=\frac{\sum_{i=j}^{n+j-1} D_{i}}{n}, \\
\overline{\phi_{I_{j}}}=\frac{\sum_{i=j}^{n+j-1} \phi_{i}}{n} .
\end{gathered}
$$

In the equations, $j \in(1, N-n+1)$.

Because the FD can reflect the complexity of the rock pore structure and the calculated porosity represents the porosity of the rock, to a certain extent, Eq. (9) was put forward to be the selecting principle. Similar to the standard deviation principle, in Eq. (9), $\sqrt{\sum_{i=1}^{N}\left(D_{i}-\overline{D_{I_{j}}}\right)^{2}} / N$ represents the dispersion degree between the FD of the combination $I_{j}$ and the global $\mathrm{FD}$, and $\sqrt{\sum_{i=1}^{N}\left(\phi_{i}-\overline{\phi_{I_{j}}}\right)^{2}} / N$ represents the dispersion degree between the porosity of the combination $I_{j}$ and the global porosity. The smaller the $\Omega\left(I_{j}\right)$, the smaller the deviation between combination $I_{j}$ and the overall data, that is, the more similar the combination $I_{j}$ is to the whole. Accordingly, by calculating the minimum value of Eq. (9), the best combination can be found. Equation (9) is as follows:

$$
\begin{aligned}
\Omega\left(I^{*}\right) & =\min \left\{\begin{array}{l}
\Omega\left(I_{j}\right) \\
= \\
\sqrt{\frac{\sum_{i=1}^{N}\left(D_{i}-\overline{D_{I_{j}}}\right)^{2}}{N}}+\sqrt{\frac{\sum_{i=1}^{N}\left(\phi_{i}-\overline{\phi_{I_{j}}}\right)^{2}}{N}} \\
\end{array}\right.
\end{aligned}
$$




$$
=\left\{\begin{array}{l}
\sqrt{\frac{\sum_{i=1}^{N}\left(D_{i}-\overline{D_{I_{1}}}\right)^{2}}{N}}+\sqrt{\frac{\sum_{i=1}^{N}\left(\phi_{i}-\overline{\phi_{I_{1}}}\right)^{2}}{N}} \\
\sqrt{\frac{\sum_{i=1}^{N}\left(D_{i}-\overline{D_{I_{2}}}\right)^{2}}{N}}+\sqrt{\frac{\sum_{i=1}^{N}\left(\phi_{i}-\overline{\phi_{I_{2}}}\right)^{2}}{N}} \\
\cdots \ldots \\
\sqrt{\frac{\sum_{i=1}^{N}\left(D_{i}-\overline{D_{I_{N-n+1}}}\right)^{2}}{N}} \\
+\sqrt{\frac{\sum_{i=1}^{N}\left(\phi_{i}-\overline{\phi_{I_{N-n+1}}}\right)^{2}}{N}}
\end{array}\right.
$$

In the equation, $j \in(1, N-n+1), I^{*}$ is the best combination, $\min ()$ is the function to find the smallest non-negative number.

Through the above steps, we finally obtained the most representative core sample by computer.

\section{APPLICATION AND DISCUSSION}

In order to verify the practicability and effectiveness of this method, a carbonate core $C^{*}$ was chosen for testing and evaluation. The petrophysical parameters of $C^{*}$ are shown in Table 1

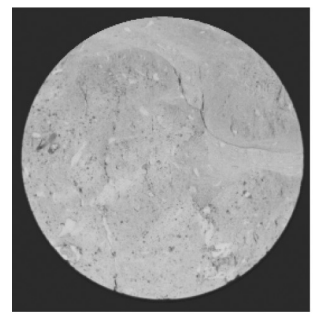

pic1

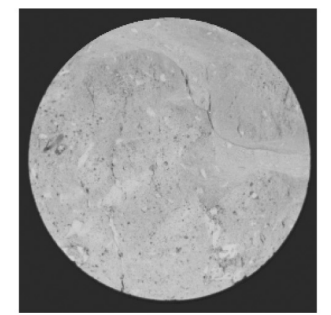

pic2

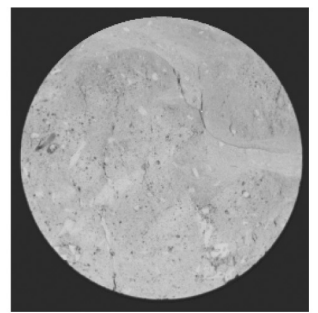

pic3
Table 1 Petrophysical Parameters of the Carbonate Core $C^{*}$ Measured by Experiment.

\begin{tabular}{cccc}
\hline $\boldsymbol{L}(\mathbf{c m})$ & $\boldsymbol{R}(\mathbf{c m})$ & $\phi(\%)$ & $\boldsymbol{K}(\mathbf{m D})$ \\
\hline 10.0 & 2.5 & 20.14 & 6.613 \\
\hline
\end{tabular}

As described in Sec. 3, the test procedure is as follows.

First, according to the flow of step 1, the core $C^{*}$ was scanned by $\mathrm{X}$-ray $\mathrm{CT}$ at a resolution of $0.1 \mathrm{~mm}$, the scan spacing $d$ was $0.1 \mathrm{~mm}, 1000$ gray scale images were obtained and stored in the scanning order, as shown in Fig. 3 .

Then 1000 gray scale images were processed according to step 2, the relevant parameters such as the FD of each image $D_{i}(i \in(1,1000))$ and the porosity of each image $\phi_{i}(i \in(1,1000))$ were calculated, as shown in Fig. 4

Finally, the procedure proceeded to step 3 , we set the number of grouping images $n$ as 300, there are 701 kinds of image combinations. The average FD $\overline{D_{I_{j}}}$ and the average porosity $\overline{\phi_{I_{j}}}$ of each combination were calculated as shown in Fig. 5, and then $\Omega\left(I_{j}\right)(j \in(1,701))$ were calculated as shown

Fig. 3 Gray scale images of core $C^{*}$.

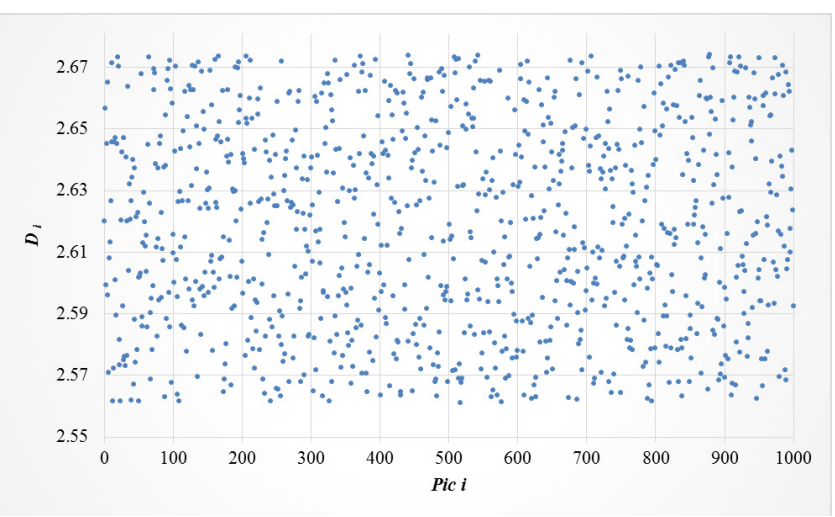

(a) FD of each image $D_{i}$

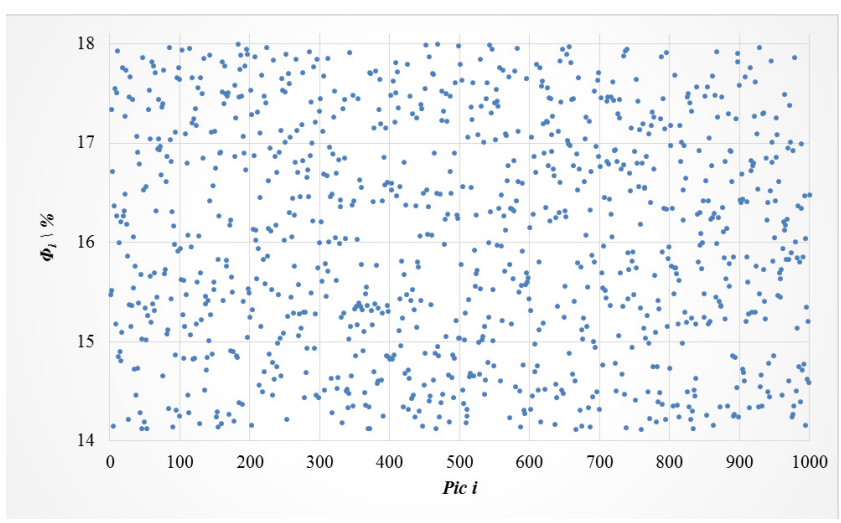

(b) Porosity of each image $\phi_{i}$

Fig. 4 Relevant parameters of 1000 gray scale images. 


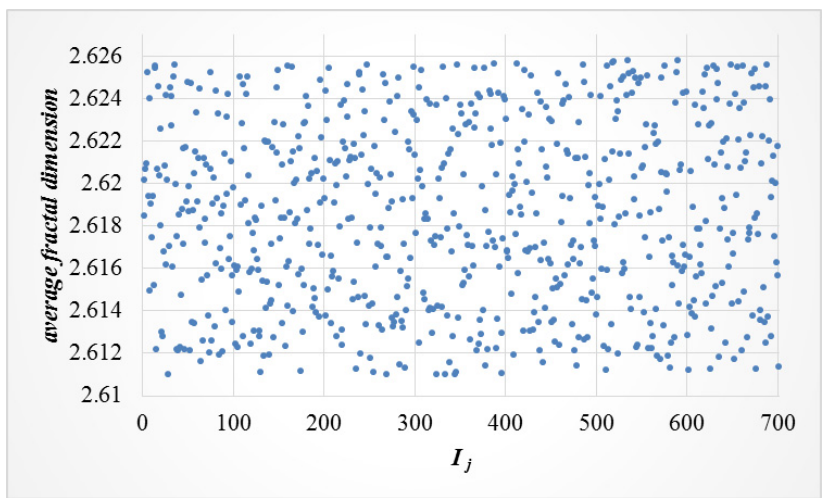

(a) Average FD $\overline{D_{I_{j}}}$ of each combination.

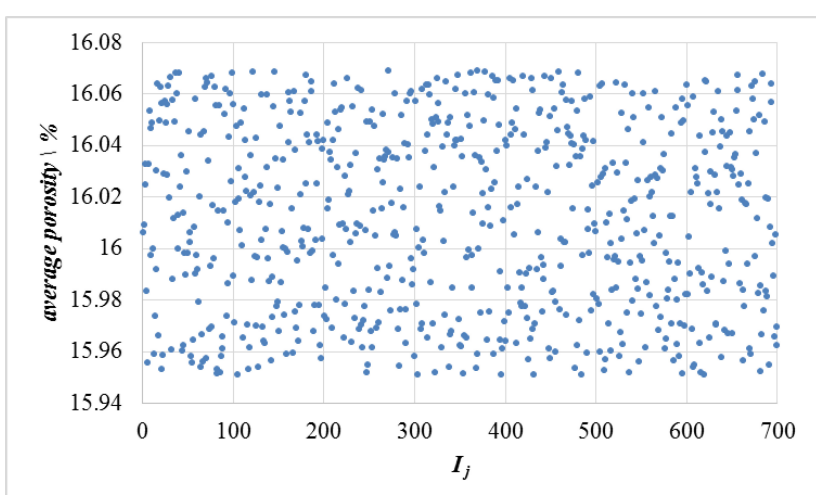

(b) Average porosity $\overline{\phi_{I_{j}}}$ of each combination.

Fig. 5 Relevant parameters of 701 combinations.

in Fig. 6 by Eq. (9)). In contrast, it was not difficult to find that $\Omega\left(I_{28}\right)$ was the minimum, so the optimal combination was determined as $I_{28}$.

Three cores $C_{28}, C_{350}$ and $C_{701}$ were cut from $C^{*}$ by measurement, where $C_{28}$ was the core segment corresponding to $I_{28}=[\operatorname{pic} 28$, pic $29, \ldots$, pic 327$]$, $C_{350}$ corresponded to $I_{350}=[$ pic350, pic351,..., pic649] and $C_{701}$ corresponded to $I_{701}=$ [pic701, pic702,..., pic1000]. The porosity and permeability

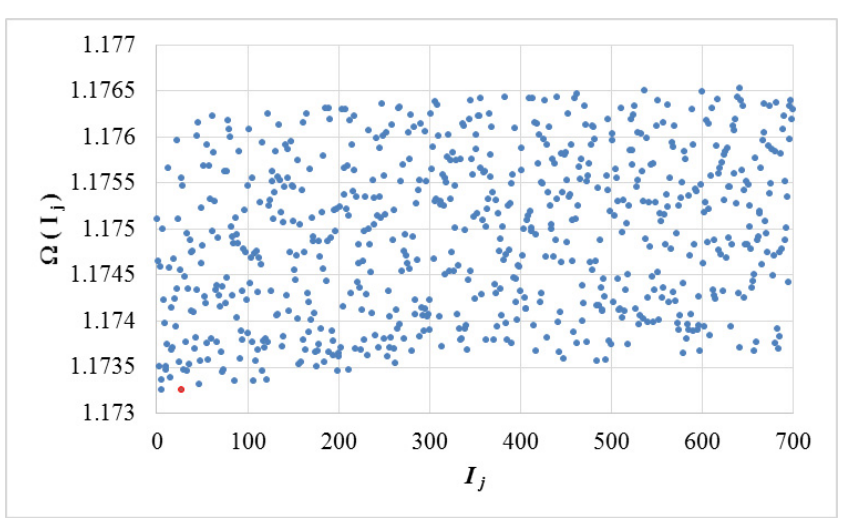

Fig. 6 Discrete values of 701 combinations.
Table 2 Petrophysical Parameters of $C^{*}, C_{28}, C_{350}$ and $C_{701}$ Measured by Experiments.

\begin{tabular}{lrccccc}
\hline & $\begin{array}{c}\boldsymbol{L} \\
(\mathbf{c m})\end{array}$ & $\begin{array}{c}\boldsymbol{R} \\
(\mathbf{c m})\end{array}$ & $\boldsymbol{\phi}(\boldsymbol{\%})$ & $\begin{array}{c}\boldsymbol{K} \\
(\mathbf{m D})\end{array}$ & $\begin{array}{c}\text { Error } \\
(\boldsymbol{\phi}) \boldsymbol{\%}\end{array}$ & $\begin{array}{c}\text { Error } \\
(\boldsymbol{K}) \boldsymbol{\%}\end{array}$ \\
\hline $\boldsymbol{C}^{*}$ & 10.0 & 2.5 & 20.14 & 6.613 & 0 & 0 \\
$\boldsymbol{C}_{\mathbf{2 8}}$ & 3.0 & 2.5 & 20.42 & 6.683 & 1.390 & 1.059 \\
$\boldsymbol{C}_{\mathbf{3 5 0}}$ & 3.0 & 2.5 & 21.51 & 7.027 & 6.802 & 6.260 \\
$\boldsymbol{C}_{\mathbf{7 0 1}}$ & 3.0 & 2.5 & 19.13 & 6.836 & 5.015 & 3.372 \\
\hline
\end{tabular}

Table 3 Discrete Values of $C_{1}$, $C_{273}$ and $C_{673}$.

\begin{tabular}{cccc}
\hline & $C_{\mathbf{2 8}}$ & $C_{\mathbf{3 5 0}}$ & $\boldsymbol{C}_{\mathbf{7 0 1}}$ \\
\hline $\boldsymbol{\Omega ( \boldsymbol { I } _ { \boldsymbol { j } } )}$ & 1.173255 & 1.175344 & 1.174669 \\
\hline
\end{tabular}

of $C_{28}, C_{350}$ and $C_{701}$ were measured, as shown in Table 2 ,

In this paper, the porosity of the rock samples is the conventional gas measurement porosity using the nitrogen test, and the permeability is the Klinkenberg permeability measured when the net confining pressure is $2 \mathrm{MPa}$. Through the analysis of Table 2, it is obvious that the porosity error and permeability error of the preferred core $C_{28}$ are the smallest and all parameters are close to those of $C^{*}$ very much; however, the subjectively selected cores $C_{350}$ and $C_{701}$ have relatively large errors compared with the core $C^{*}$. On the one hand, the experimental results show that the selected core $C_{28}$ has porosity and permeability similar to that of long core $C^{*}$, and to a certain extent, it demonstrates that the core $C_{28}$ is representative and proves that the method is feasible and practical. On the other hand, it also certifies that the petrophysical parameters of the subjectively selected rock samples may vary considerably from the whole rock.

To further verify the validity of this method, the discrete values of $C_{350}$ and $C_{701}$ were calculated by Eq. (9), as shown in Table 3 . Combined with Tables 2 and 3, it can be drawn that the smaller the discrete value $\Omega\left(I_{j}\right)$, the smaller the porosity error and permeability error. So it proves that the proposed criterion, Eq. (9), is valid and can be used to be the selecting principle.

\section{CONCLUSION}

In conclusion, a facile approach to selecting representative rock samples in digital core modeling is reported. Based on fractal theory and computer technology, with the help of X-ray CT scan, the 
interesting core column is scanned and CT scan images are grouped by our proposed sequence. Then, the FD of each image $D_{i}$, the porosity of each image $\phi_{i}$, as well as the average $\mathrm{FD} \overline{D_{I_{j}}}$ and the average porosity $\overline{\phi_{I_{j}}}$ of each combination are calculated. According to our proposed criteria as Eq. (9), the best combination is selected and the representative core is obtained. It demonstrates that the selected core is representative and proves that the method is feasible and practical through a simple experiment. The novelty of this paper is to propose an objective method to select representative rock samples combined with fractal theory which can avoid some subjective errors and make some contribution to the rock numerical simulation. However, there may be some imperfections and limitations, such as the high cost of CT scan, and the lack of a large number of experiments to verify the reliability of this method and so on. At the same time, this method can be applied not only to digital cores, but also to other areas of sample selection.

\section{ACKNOWLEDGMENTS}

We gratefully acknowledge financial support from the National Science and Technology Major Project (2017ZX05013-001).

\section{REFERENCES}

1. C. H. Arns, F. Bauget, A. Limaye et al., Pore-scale characterization of carbonates using X-ray microtomography, SPE J. 10(4) (2005) 475-484.

2. B. Biswal, C. Manwart, R. Hilfer et al., Quantitative analysis of experimental and synthetic microstructures for sedimentary rock, Phys. A 273(3) (1999) $452-475$.

3. M. Iovea, G. Oaie, C. Ricman et al., Dual-energy $\mathrm{X}$-ray computer axial tomography and digital radiography investigation of cores and other objects of geological interest, Eng. Geol. 103(3-4) (2009) 119126.

4. J. Yao, C. C. Wang, Y. F. Yang et al., A new method for rock core characterization in sandy conglomerate media, J. Rock Soil Mech. 33(S2) (2012) 205-208.

5. D. P. Lmberopoulos and A. C. Payatakes, Derivation of topological, geometrical and correlational properties of porous media from pore-chart analysis of serial section data, J. Colloid Interf. Sci. 150(1) (1992) 61-80.

6. L. Tomutsa, D. B. Silin and V. Radmilovic, Analysis of chalk petrophysical properties by means of submicron-scale pore imaging and modeling, SPE Res. Eval. Eng. 10(3) (2007) 285-293.
7. H. Okabe and M. J. Blunt, Pore space reconstruction using multiple-point statistics, J. Petrol. Sci. Eng. 46(1-2) (2005) 121-137.

8. H. J. Vogel and K. Roth, Quantitative morphology and network representation of soil pore structure, Adv. Water Res. 24(3-4) (2001) 233-242.

9. J. T. Fredrich, B. Menendez and T. F. Wong, Imaging the pore structure of geomaterials, Science 268(5208) (1995) 276-279.

10. H. Westphal, I. Surholt, C. Kiesl et al., NMR measurements in carbonate rocks: Problems and an approach to a solution, Pure Appl. Geophys. 162(3) (2005) 549-570.

11. C. H. Arns, The Influence of Morphology on Physical Properties of Reservoir Rocks (The University of New South Wales, Sydney, 2002).

12. J. Coenen, E. Tchouparova and X. Jing, Measurement parameters and resolution aspects of micro X-ray tomography for advanced core analysis, Proceedings of International Symposium of the Society of Core Analysts (2004), Abu Dhabi, UAE.

13. P. E. Aren and S. Bakke, Process based reconstruction of sandstones and prediction of transport properties, Transp. Porous Media 46(2-3) (2002) 311-343.

14. M. Joshi, A Class of Stochastic Models for Porous Media (University of Kansas, Kansas, 1974).

15. R. D. Hazlett, Statistical characterization and stochastic modeling of pore networks in relation to fluid flow, Math. Geol. 29(6) (1997) 801.

16. C. H. Arns, F. Bauget, A. Limaye et al., Pore-scale characterization of carbonates using X-ray microtomography, SPE J. 10(4) (2005) 475-484.

17. L. M. Keller, P. Schuetz, R. Erni et al., Characterization of multi-scale microstructural features in Opalinus Clay, Micropor. Mesopor. Mater. 170 (2013) 83-94.

18. D. Wildenschild and A. P. Sheppard, X-ray imaging and analysis techniques for quantifying porescale structure and processes in subsurface porous medium systems, Adv. Water Res. 51(1) (2013) 217-246.

19. M. J. Blunt, B. Bijeljic, H. Dong et al., Porescale imaging and modeling, Adv. Water Res. 51(1) (2013) 197-216.

20. J. Feder, Fractals (Plenum Press, New York, 1988).

21. B. M. Yu and P. Cheng, A fractal model for permeability of bi-dispersed porous media, Int. J. Heat Mass Transf. 45(14) (2002) 2983-2993.

22. B. B. Mandelbrot, The Fractal Geometry of Nature (Freeman, San Francisco, 1983).

23. J. C. Cai, B. M. Yu, M. Q. Zou et al., Fractal characterization of spontaneous co-current imbibition in porous media, Energy Fuels 24(3) (2010) 1860-1867.

24. B. M. Yu and J. H. Li, Some fractal characters of porous media, Fractals 9(3) (2001) 365-372. 
25. J. C. Cai, B. M. Yu, M. Q. Zou et al., Fractal analysis of invasion depth of extraneous fluids in porous media, Chem. Eng. Sci. 65(18) (2010) 5178 5186.

26. B. M. Yu, Analysis of flow in fractal porous media, Appl. Mech. Rev. 61(5) (2008) 1-19.

27. J. C. Cai and B. M. Yu, Prediction of maximum pore size of porous media based on fractal geometry, Fractals 18(4) (2010) 417-423.

28. P. Xu, A discussion on fractal models for transport physics of porous media, Fractals 23(3) (2015) 1530001.
29. B. M. Yu, Fractal dimensions for multiphase fractal media, Fractals 14(2) (2006) 111-118.

30. P. Xu and B. M. Yu, Developing a new form of permeability and Kozeny-Carman constant for homogeneous porous media by means of fractal geometry, Adv. Water Res. 31(1) (2008) 74-81.

31. Y. J. Liu, B. M. Yu, P. Xu et al., Study of the effect of capillary pressure on permeability, Fractals 15(1) (2007) 55-62.

32. X. C. Jin, S. H. Ong and Jayasooriah, A practical method for estimating fractal dimension, Patt. Recogn. Lett. 16(1995) (1995) 457-464. 